\title{
Digital scriptum - novel approach of student experiment preparation
}

\author{
Roß, Nikolas ${ }^{\mathrm{a}}$; Kuska, Robert ${ }^{\mathrm{a}}$; Rieth, Iris ${ }^{\mathrm{b}}$ and Frerich, Sulamith ${ }^{\mathrm{a}}$ \\ ${ }^{a}$ Virtualisation of Process Technology, Department of Mechanical Engineering, Ruhr- \\ University Bochum, Germany, 'Fluid Separations, Department of Mechanical Engineering, \\ Ruhr-University Bochum, Germany
}

\begin{abstract}
In mechanical engineering, students pass several seminars aiming at selfconducted experiments and trials. In the ELLI project (Excellent Teaching and Learning in Engineering Science), different virtual and remote laboratories have been installed within the three engineering departments at the Ruhr-University Bochum. The preparation in a classic experiment is usually done with a classic paper print scriptum. This is not suitable for a virtual or remote laboratory, since, amongst others, no supervision is provided. Due to that, a classic scriptum has been transferred in a digital scriptum based on the Open Source software "Adapt Learning" and has been extended by various implemented digital items. This contribution is discussing preliminary student experiences and identified prospective improvements. It turns out that the digital scriptum is suitable for the preparation of virtual and remote laboratories but can be an excellent alternative to the classic printout scriptum in a student experiment in a hands on laboratory as well.
\end{abstract}

Keywords: Digital scriptum; preparation; student experiments; remote laboratories; mechanical engineering; engineering education 


\section{Introduction}

Project ELLI (Excellent Teaching and Learning in Engineering Science) is a joint project of the three German universities RWTH Aachen, Ruhr-University Bochum and TU Dortmund University, and it was funded by the Federal Ministry of Education and Research. Considering teachers' and learners' perspectives, the project aims at improving existing concepts in higher engineering education, and the development of new and innovative approaches is pursued. In the past years, a pool of remote and virtual laboratories has been set up in order to gain flexibility in the usage of experimental equipment in different scenarios. At Ruhr-University Bochum, these laboratories are maintained from all engineering faculties, so topics from environmental, mechanical and electrical engineering are covered.

Since practical experience is considered as essential part in engineering education, engineering students are usually encouraged to take part in seminars aiming at selfconducted experiments and trials. Thus, increasing numbers in study programs are a challenge for providing adequate hands-on laboratories. Currently, it is planned to implement the virtual and remote laboratories at Ruhr-University Bochum as digital enhancement of seminars aiming at practical experiences. The preparation of students prior to conducting an experiment is essential for the learning outcome of both digital and handson laboratories. Therefore, the aim of this contribution is showing first experiences of student preparation for conducting a digital experiment in a remote laboratory.

\section{Student experiment preparation}

The student introduction into experimental set-ups and exercises using virtual or remote equipment differs strongly from classical preparation in laboratories. Usually, before gaining access to a hands-on laboratory, students are preparing themselves, using scriptums, textbooks or databases.

\subsection{Classic scriptum}

A representative execution of a hands-on laboratory by students at the Ruhr-University Bochum begins with the preparation of the topic. The main and only relevant object is a classic scriptum, whose content leans on the respective lectures teaching this topic. Its structure usually aims at the first parts of typical journal articles:

- Introduction

- Materials and Methods

- Theoretical background

- Experimental set-up and execution 
The pdf-file scriptum can be printed out. It contains fixed content, and its continuous text is mostly interrupted by specific and explanatory figures and tables. This is completed by issues and tasks the students have to solve and by information on how they have to handle the gained data.

The preparation is followed by a person-to-person assessment of the laboratories' supervisor. A group of up to four students receive random questions, which can be answered by the knowledge gained in the classic scriptum. The assessment mainly aims at a validation of the students' preparation in order to verify their ability to undertake this experiment.

\subsection{Interactive scriptum}

In the last decades, a trend on remotely accessible real and virtual laboratories was oberserved. While the technology improved, companies and universities realized the potential in productivity, availability and education. Increasing internet speed and computational power lead to a variety of approaches, but the goals in engineering education are still the same: Students should know the handling of machines and processes, and they are able to understand theoretical backgrounds by applying it in practical work. Although personal feedback is crucial for the students' learning-by-doing, the supervision of experimental performances is not given in remote access. This change to unsupervised experiments results in different learning outcomes (Lindsay \& Good, 2005). Two groups of students were observed, and the group with access to the real laboratory performed better (Lindsay \& Good, 2005). Thus it was shown that a better understanding of the apparatus before execution a remote experiment is helpful. Still, the students' preparation is neglected by the development. Hence, the classic scriptum needs to be adjusted to the still ongoing evolution of digitalized laboratory education to enhance the students' cognition of the laboratory.

This work deals with the realization of a modern, flexible, intuitive and interactive scriptum. It should be accessible from all devices, online, offline and be able to use digital items like interactive figures and animations that a classic scriptum cannot. Furthermore, it should give students a variety of possible preparation ways, so that the system aligns more to the students' learning preferences.

Different approaches and software systems can be found that are applicable in the field of education. The preparation can be switched to videos or to an online survey (LinkedIn Austria GmbH, 2018; Netigate AB, 2018). As well flexible systems can switch between informing and testing via questions or similar with lots of possibilities (Elucidat Limited, 2018; Strategic Technology Solutions dba SmartBuilder, 2018). 
While the aforementioned solutions are either limited in their functionality or are commercial, "Adapt Learning" is a freeware Open Source authoring tool with some significant benefits (Adapt community, 2018).

It uses HTML5 to achieve a browser-based experience and has a responsive design that allows the accessibility and readability on all kind of devices and operating systems. In addition it is Open Source, so it can be extended. A community develops plugins which can be implemented in the own system. In Adapt Learning many digital items are already included, such as video implementation, interactive figures and assessments with variable question types. An important maintenance factor is the easy to use graphical user interface.

However, what is possible in a modern scriptum? Primary the classic scriptum is represented in a new way. Then some digital items can be implemented where a benefit is to be foreseen, such as interactive figures to introduce the experimental set-up. Questions for self-reflection can be implemented after each chapter. A feedback system for wrong answers can lead to the important passage within the scriptum and offer hints to find deeper understanding online or in books. Video or animation implementation can be used to visualize problems, which are hard to describe with words. Complex systems can be easier to access with 3D models instead of static pictures with various angles of the apparatus. Interactive graphs and charts can be used by the students to expand the knowledge of some behavior of the system by easily zoom in and out and concatenate various information autonomously. A final assessment also helps to self-reflect the own knowledge. Where possible, gamification elements can be implemented to already train the student for the performance (Kruse et al., 2018). The participating students should feel well prepared, so no supervisor is necessary in the execution of the experiment.

In this work, a first draft of the new scriptum was realized on a real laboratory, which can be performed via remote access or as a hands-on laboratory. By that, the suitability of the scriptum can be verified for both cases.

\section{Experiment set-up}

The considered experimental set-up was developed for teaching the principle of heat transfer in the context of a student laboratory. The experimental set-up is integrated in the remote laboratory platform "ELLI Lab Library” to enable online operated measurements (Kruse et. al., 2016). The goal is to comprehend the temperature profile in a heat exchanger using a Fibre Brag Grating Sensor as measurement technique. The students should determine the Nusselt number and heat transfer coefficient as typical key figures, for this heating system and compare it to data from literature. 


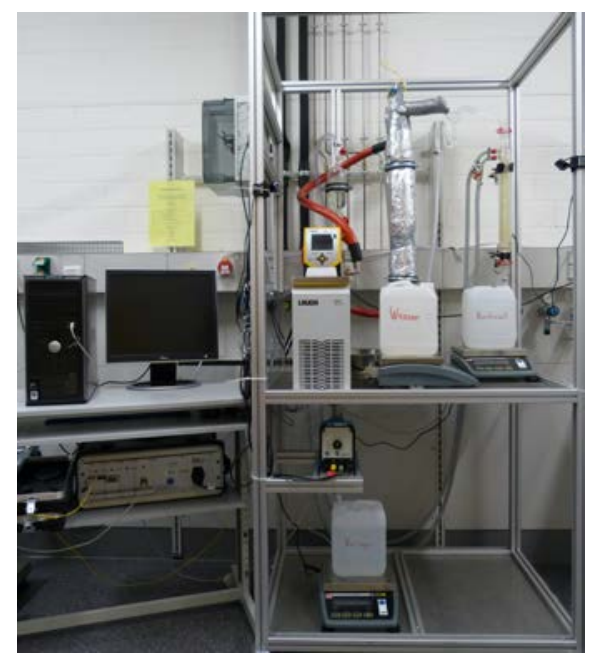

Figure 1: Experimental set-up

The main part of the experimental set-up in Figure 1 is the double-walled heat exchanger with the installed Fibre Brag Grating Sensor for temperature measurements. This minimally-invasive measurement technique allows the determination of the temperature of the process medium in the heat exchanger at 13 axial positions (Stegehake \& Grünewald, 2017). While the heating fluid flows downwards in the jacket, the process medium gets pumped through the inlet of the heat exchanger upwards (counter flow). In order to measure the mass flow of the inlet process medium and the outcoming liquid and vaporized parts of it, there are three weighing instruments.

The thermostat and the pump can be regulated manually at the device itself, but the regulation can also be done in a software for controlling processes. LabVIEW is used for controlling pump and thermostat, and it shows the temperature of the heating fluid, the mass in the collecting containers and the measured temperatures inside the heat exchanger. The students can observe the separation of the liquid and vaporized part of the outcoming process medium via camera, which is also integrated in LabVIEW. The communication between the students and the laboratory is realized by iLab, an Open Source time management system. It allows students to access the experiment, read off the measured data and regulate the pump and thermostat online.

Before the students start the remote laboratory, they need to do the online scriptum. This scriptum has the challenge to impart the experimental set-up and the function of its components without the students seeing it live. The tutorial focuses on understanding the principle of a heat exchanger, the online handling of this experimental set-up and the collection of all relevant experimental data. When the laboratory is started, the students have to heat up the thermostat first and to choose set points of the thermostat and pump 
inside a given range, which is integrated in LabVIEW to ensure a safe operation. If the heat exchanger reaches stationary conditions for the chosen set point, the students have to read off the temperatures inside the heat exchanger and the weight of the collected samples of the processed medium at three points in time. After the students finished the remote laboratory, the calculation of the key figures has to be done, using the correlations and data, which are given on the online platform.

\section{Results and discussion}

The developed digital scriptum is divided in different chapters as shown in Figure 2. The start screen includes the main information like topic, contact person and experimental location. By using the "Start"-button, the preparation is started and the view changes to the chapter overview. This first approach of preparation is separated like the classic scriptum in six main chapters. In each chapter, different kinds of presentations, questions or assessment components were used to allow interactions either to inform the students or to enable their self-reflection in study progress.

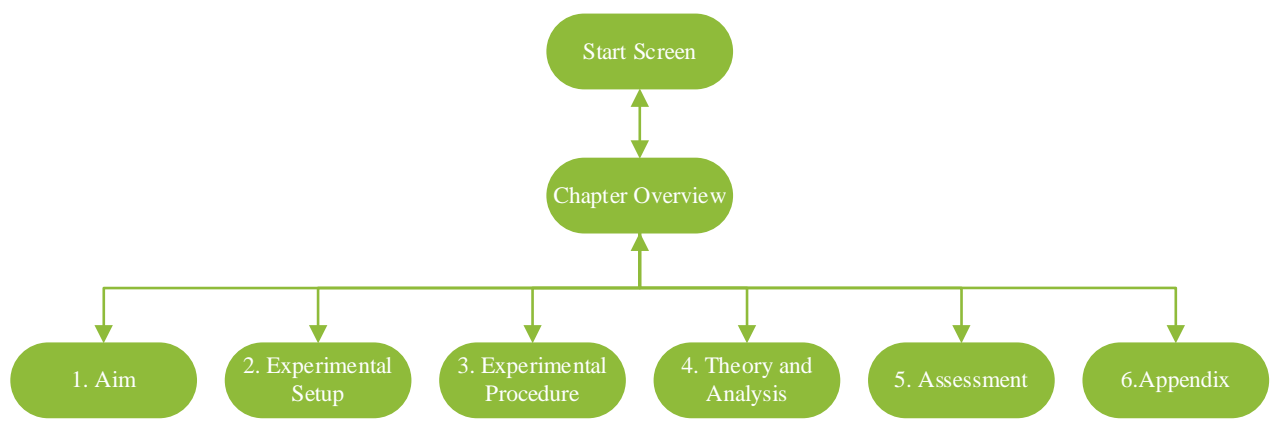

Figure 2: Structure of the digital scriptum

To collect first experiences, a testing of the digital scriptum was done at the RuhrUniversity Bochum with a mixed focus group. The focus group contained 15 students out of different engineering science courses (Mechanical Engineering, Environmental Technology and Resource Management) with different progress of their studies (Bachelor/Master/Doctorate). The laboratory preparation was installed offline on surface tablets. The students could freely choose the orientation of the tablets, concerning the responsive design of Adapt Learning. The preparation with the digital scriptum took the students around 20 minutes. Afterwards, they were asked to fill out a questionnaire to collect the student's impressions.

The testing showed that the students especially liked the interactive introducing figure for the experimental set-up. The implemented set-up is shown on the left side in Figure 3. Via icons, the students were able to inform themselves about each specific item of the 
processing plant. As shown on the right side in Figure 3, they get detailed information by activating the specific icon on the picture.
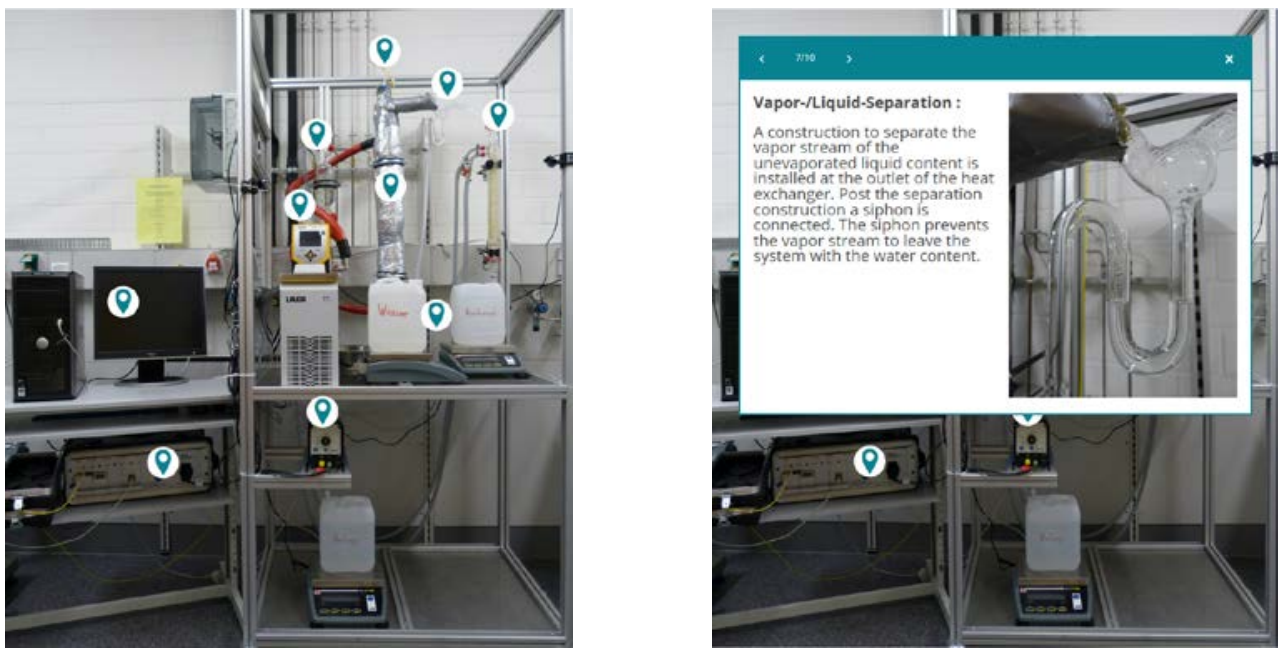

Figure 3: Experimental set-up (left) implemented in Adapt Learning and pop-ups with detailed information for each marked part (right)

Another noted positive aspect was the structure of the scriptum itself as it is visualized in Figure 4. Since the structure of the digital scriptum was oriented in a similar way like the classic scriptum, the students had no orientation problems.
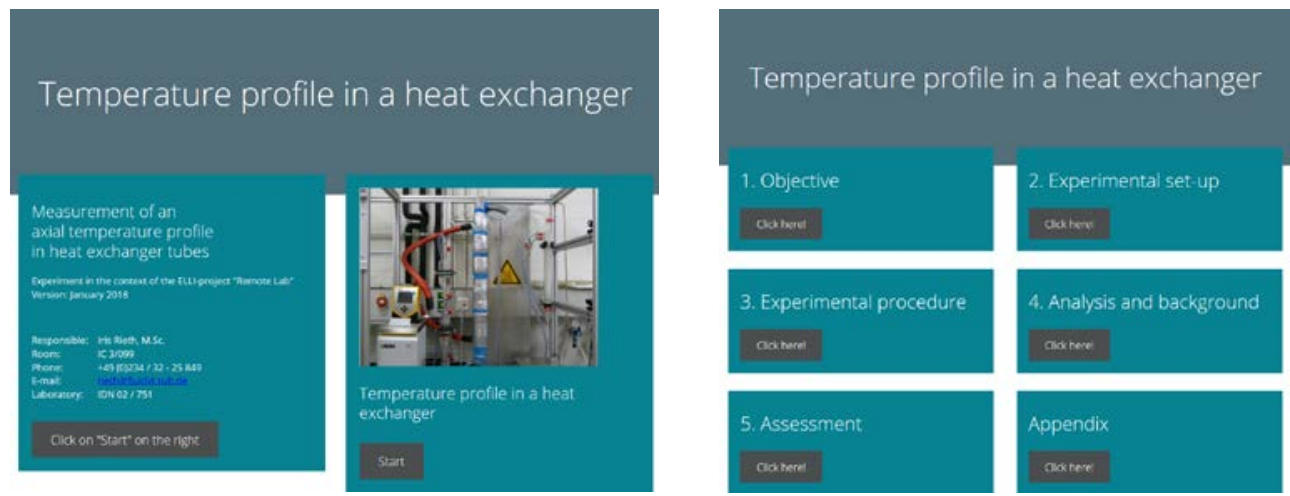

Figure 4: Start screen (left) and chapter overview (right) of the digital scriptum

The handling of the preparation tool was also noted positively. The students needed no further explanation about the tool, thus they could prepare themselves without further assistance.

As negative feedback, the students would have favored more content and explanations especially reagarding the innovative temperature measuring system. Furthermore, the 
students asked for complementary elements like calculation examples, videos of the running experiment or explanatory videos of the inner side of the heat exchanger. It shows a general interest in the possibilities of the innovative way of laboratory preparation, which will be considered in future steps of development.

\section{Summary and outlook}

The first trial showed that the digital scriptum could be an excellent alternative to the classic printout scriptum. The students can inform, interact and self-reflect themselves, which is usually not possible in this extent by using a classic scriptum. The laboratory and its preparation should be tested and evaluated with a bigger, more homogeneous group in the context of a practical course, in order to gain further insight. In the prospective, the scriptum can be supplemented by further elements like interactive diagrams, videos or stream animations to visualize problems. The final assessment cannot only help the students to self-reflect their knowledge, a successful completion can be used to grant the students the direct online access to the remote laboratory in the future.

\section{References}

Adapt community. (2018). Adapt Learning. Retrieved from https://www.adaptlearning.org/

Elucidat Limited. (2018). Elucidat. Retrieved from https://www.elucidat.com/

Kruse, D., Frerich, S., Petermann, M., Ortelt, T. R., \& Tekkaya, A. E. (2016). Remote labs in ELLI: Lab experience for every student with two different approaches. In Proceedings of 2016 IEEE Global EDUCON: Date and venue: 10-13 April 2016, Abu Dhabi, UAE (pp. 469-475). https://doi.org/10.1109/EDUCON.2016.7474595

Kruse, D., Kuska, R., Frerich, S., May, D., Ortelt, T. R., \& Tekkaya, A. E. (2018). More Than “Did You Read the Script?”. In M. E. Auer \& D. G. Zutin (Eds.), REV 2017, held 15-17 March 2017, Columbia University, New York, USA (Vol. 22, pp. 160-169). https://doi.org/10.1007/978-3-319-64352-6_16

Lindsay, E. D., \& Good, M. C. (2005). Effects of Laboratory Access Modes Upon Learning Outcomes. IEEE Transactions on Education, 48(4), 619-631. https://doi.org/10.1109/TE.2005.852591

LinkedIn Austria GmbH. (2018). video2brain. Retrieved from https://www.video2brain.com/

Netigate AB. (2018). Netigate. Retrieved from https://www.netigate.net/

Stegehake, C., \& Grünewald, M. (2017). Anwendung von faseroptischer Messtechnik zur gering-invasiven Temperaturprofilmessung in Reaktoren. Chemie Ingenieur Technik, 89(4), 480-485. https://doi.org/10.1002/cite.201600138

Strategic Technology Solutions dba SmartBuilder. (2018). smartbuilder. Retrieved from http://www.smartbuilder.com/ 